\title{
Wannier-function-based constrained DFT with nonorthogonality-correcting Pulay forces in application to the reorganization effects in graphene-adsorbed pentacene
}

\author{
Subhayan Roychoudhury, David D. O’Regan, ${ }^{*}$ and Stefano Sanvito \\ School of Physics, AMBER and CRANN Institute, Trinity College Dublin, Dublin 2, Ireland
}

(Received 5 February 2018; published 14 May 2018)

\begin{abstract}
Pulay terms arise in the Hellmann-Feynman forces in electronic-structure calculations when one employs a basis set made of localized orbitals that move with their host atoms. If the total energy of the system depends on a subspace population defined in terms of the localized orbitals across multiple atoms, then unconventional Pulay terms will emerge due to the variation of the orbital nonorthogonality with ionic translation. Here, we derive the required exact expressions for such terms, which cannot be eliminated by orbital orthonormalization. We have implemented these corrected ionic forces within the linear-scaling density functional theory (DFT) package ONETEP, and we have used constrained DFT to calculate the reorganization energy of a pentacene molecule adsorbed on a graphene flake. The calculations are performed by including ensemble DFT, corrections for periodic boundary conditions, and empirical Van der Waals interactions. For this system we find that tensorially invariant population analysis yields an adsorbate subspace population that is very close to integer-valued when based upon nonorthogonal Wannier functions, and also but less precisely so when using pseudoatomic functions. Thus, orbitals can provide a very effective population analysis for constrained DFT. Our calculations show that the reorganization energy of the adsorbed pentacene is typically lower than that of pentacene in the gas phase. We attribute this effect to steric hindrance.
\end{abstract}

DOI: 10.1103/PhysRevB.97.205120

\section{INTRODUCTION}

Across a wide range of electronic-structure theory methods, such as constrained density functional theory (cDFT) [1,2], density functional theory plus Hubbard $U(\mathrm{DFT}+U)[3,4]$, DFT combined with dynamical mean-field theory (DMFT) [5,6], wave-function embedding [7,8], and some perturbative approaches in quantum chemistry [9], the population of a particular subspace is physically relevant and the total energy depends explicitly upon it. Thus, the ability to define appropriate subspaces for population analysis is of considerable importance. This is exemplified by sustained efforts in recent years in the development of physically motivated orbitals such as maximally localized Wannier functions (MLWFs) [10], nonorthogonal localized molecular orbitals (NOLMOs) [11], muffin-tin orbitals (MTOs) [12], and natural bond orbitals (NBOs) [13] for use in system-dependent, adaptive population analysis. Population analysis by means of projection of orbitalbased subspaces has attracted detailed investigation in recent years [14,15]; in particular, the effects of projector orbital ambiguity in DFT $+U[16,17]$ and DFT+DMFT [18] have been investigated in detail.

In calculations in which the total energy depends explicitly upon localized orbitals that are centered on atoms, Pulay terms $[19,20]$ arise in the Hellmann-Feynman forces due to spatial translations of the orbitals. It is, however, less known, although previously identified $[21,22]$, that additional Pulay terms emerge when the total energy also depends on the overlap matrix of such orbitals. This is necessary for correct

*david.o.regan@tcd.ie population analysis using nonorthogonal orbitals. In fact, these forces are present for any multicenter atomic projection of the density or the Kohn-Sham density matrix. They exist when using orthonormal orbitals such as MLWFs, for example, since any ionic movement typically breaks the orthonormality. Thus, unless the forces take into account that the orbitals are regenerated or orthonormalized following a translation, a condition that is difficult to encode, then unconventional nonorthogonality Pulay forces arise even for orbitals that are defined as orthonormal.

Approaches for calculating the necessary corrections, based on a Löwdin orthonormalized representation of the subspace projection, invariably encounter a cumbersome, difficult to solve, Sylvester equation [23] of the form

$$
\begin{aligned}
\frac{d \mathbf{O}}{d R_{i}} & =\mathbf{O}^{1 / 2} \frac{d \mathbf{O}^{1 / 2}}{d R_{i}}+\frac{d \mathbf{O}^{1 / 2}}{d R_{i}} \mathbf{O}^{1 / 2} \text { or } \\
-\frac{d \mathbf{O}}{d R_{i}} & =\mathbf{O}^{1 / 2} \frac{d \mathbf{O}^{-1 / 2}}{d R_{i}}+\frac{d \mathbf{O}^{-1 / 2}}{d R_{i}} \mathbf{O}^{1 / 2},
\end{aligned}
$$

where $\mathbf{O}$ is the projector orbital overlap matrix and $R_{i}$ is a Cartesian component of the ionic position. Here the solution for $\mathbf{O}^{1 / 2}$ is required. An approximate method for working around this problem, based on neglecting off-diagonal matrix elements in $\mathbf{O}^{-1 / 2}$, has been recently proposed in Ref. [22]. Reference [21] instead provides a formula for the full matrix $d \mathbf{O}^{1 / 2} / d R_{i}$, which makes use of the basis of the shared eigenvectors of $\mathbf{O}$ and $\mathbf{O}^{1 / 2}$. This necessitates matrix diagonalization. The applicability and practicality of these two approaches depend on the details of the force calculations to be undertaken.

In this work, we use nonorthogonal basis functions and their appropriate tensor notation following a long-standing tradition 
in electronic-structure theory [24-29]. We furthermore use the modern tensorially invariant population analysis [30], which has appeared in various contexts $[14,15]$ including that of cDFT [31,32]. We extend this to calculate an exact, simple, and intuitive expression for the nonorthogonality Pulay forces, which circumvents orbital orthonormalization and overlap matrix diagonalization entirely. This expression is applicable to real- and complex-valued orbitals alike, and whether or not they are orthonormal at the point of force evaluation. Avoiding matrix diagonalization ensures its applicability to large systems using linear-scaling DFT. We put our scheme to the test here by calculating the reorganization energy of a pentacene molecule physisorbed on a graphene sheet.

The paper is organized as follows. In the next section, we will define the physical problem addressed by our work, namely the calculation of the energies needed for extracting the reorganization energy of a molecular absorbate on a metallic substrate. Then we will describe our computational methods, focusing on the derivation of the forces in orbital-based cDFT, the performance of orbital-based population analysis, and a number of practical considerations addressed using the ONETEP code. Our results for pentacene on graphene will be presented next, followed by our conclusions.

\section{PHYSICAL PROBLEM: REORGANIZATION OF A CHARGED MOLECULE PHYSISORBED ON A METALLIC SURFACE}

The reorganization energy holds paramount importance in charge-transport calculations. Semiclassical Marcus theory [33] at high temperature, $T$, computes the probability per unit time of an electron hopping, $k_{\mathrm{ET}}$, from Fermi's golden rule as $[34,35]$

$$
k_{\mathrm{ET}}=\frac{|\langle i|\hat{H}| f\rangle|^{2}}{\hbar} \sqrt{\frac{\pi}{\lambda k_{B} T}} \exp \left[-\frac{\left(\lambda+\Delta G^{0}\right)^{2}}{4 \lambda k_{\mathrm{B}} T}\right],
$$

where $\hat{H}$ is the Hamiltonian, $|i\rangle$ and $|f\rangle$ are the initial and final electronic states, respectively, and $\Delta G^{0}$ is the change in Gibbs' free energy associated with the charge-transfer process. The reorganization energy, which enters the exponential term defining $k_{\mathrm{ET}}$, is thus an important ingredient [36-38] for the calculation of the charge hopping. In this work, we compute the reorganization energy of a pentacene molecule. In its crystalline solid-state form, pentacene is a p-type semiconductor [39] with a high hole mobility [40]. Thus, ionization reorganization effects in pentacene-based systems are of significant interest, being the subject of several theoretical and experimental studies [41-43].

Let us define the reorganization energy precisely. The ionic coordinates of any system depend on its electronic occupation. For instance, if an electron is removed from a neutral molecule, such as in photoemission spectroscopy, its ionic coordinates will readjust to a new geometry due to the local electron-phonon coupling [44,45]. Figure 1 shows two parabolic curves that represent the energy surface of the neutral molecule and that of the singly ionized one, as a function of some collective atomic coordinates. We define as $\lambda^{0}$ the energy difference between the ground-state geometry and the ground-state geometry of the charged configuration [46] when the molecule is neutral. In contrast, $\lambda^{+}$is the same quantity but

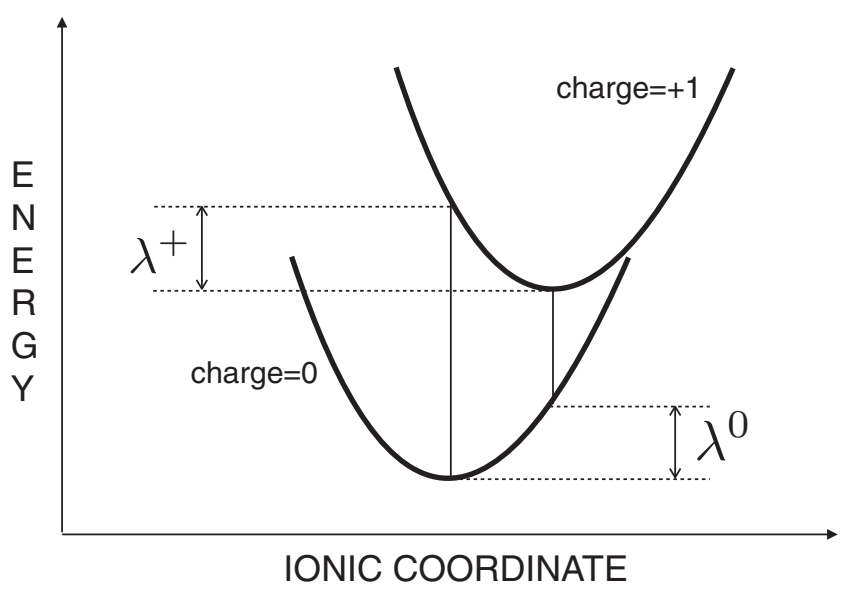

FIG. 1. Schematic diagram of the energy as a function of the ionic coordinates for a charged and a neutral molecule. The reorganization energy is defined as $\lambda^{0}+\lambda^{+}$.

calculated for the ionized system. The reorganization energy, $\lambda$, for the molecule undergoing electron removal is defined as

$$
\lambda=\lambda^{0}+\lambda^{+}
$$

where similar definitions can be given for the case in which the molecule receives an extra electron.

Theoretical approaches to compute the reorganization energy typically consist of either calculating the energy difference from the adiabatic potential energy surface, or of indirectly evaluating the molecule's normal modes [47]. Here we adopt the former approach. For an isolated pentacene molecule, an electron removal can be simulated with unconstrained DFT, and therefore it does not require the aforementioned force terms. However, this approach is not viable for the study of reorganization in systems relevant to organic semiconductor devices, where organic molecules are typically adsorbed on metallic electrodes. When a molecule is adsorbed on a metallic substrate and its highest occupied molecular orbital (HOMO) lies below the Fermi level, the hole must be prevented from migrating to the energetically favorable location of the substrate. We achieve this by using cDFT to force the hole onto the adsorbate.

cDFT has been widely applied to the study of charge transfer in organic compounds [48-56]. Recently, cDFT has been used to estimate charge-transfer excitations in bulk pentacene in the infinite-crystal limit [31]. The present work utilizes the same underlying linear-scaling cDFT implementation, itself an extension of a linear-scaling implementation of DFT $+U$ [57] using nonorthogonal generalized Wannier functions. Also relevant to this work is that cDFT has been used to simulate the removal or addition of electrons from an adsorbed molecule in the context of calculating charge-transfer energies [58,59]. Here we use cDFT in conjunction with nonorthogonality Pulay forces to calculate the reorganization energy of a pentacene molecule physisorbed on a flake of graphene. The energy of a system as a function of its geometry can contain multiple local minima, and this is particularly the case for the incommensurate corrugated system at hand. The proposed method, in conjunction with efficient sampling techniques such as 
simulated annealing [60], basin hopping [61], etc., could be used to explore such energy landscapes in the presence of orbital-based constraints. We note that a more complex system, consisting of a film of weakly bound pentacene molecules adsorbed on highly oriented pyrolytic graphite (HOPG), has been the subject of several theoretical and experimental studies [62-64]. It has been shown, in the experimental work of Ref. [62], that the reorganization energy of pentacene is there, remarkably, higher than that in the gas phase.

\section{THEORETICAL PROBLEM: POPULATION ANALYSIS AND FORCES BASED ON NONORTHOGONAL ORBITALS}

In cDFT, to date, real-space partitioning has prevailed over orbital-based population analysis methods. Central to the viability of using more chemically motivated orbitals to define the constrained population in cDFT, and perhaps hindering their adoption, is the proper treatment of their nonorthogonality. In particular, historically there has been some uncertainty $[65,66]$ as to how subspace populations should be defined in terms of nonorthogonal orbitals, which typically (but not necessarily by any means) form a subset of the basis set for the Kohn-Sham states. This uncertainty has previously been conclusively resolved within the context of $\mathrm{DFT}+U$ [30], and the correct procedure has recently been pioneered in cDFT for calculating charge-transfer energies in solid pentacene [31]. We will numerically investigate the performance of this tensorially consistent procedure for cDFT in the present work. A separate problem, which we also will touch upon in this work, is the arbitrary choice of the underlying projection orbitals in terms of their particular spatial profile.

The canonical orbital-based population analyses in quantum chemistry are due to Löwdin and Mulliken, and these are both unsuitable for cDFT. cDFT population analyses based on orbitals that are globally Löwdin orthonormalized, meaning that the entire basis set is orthonormalized before a subset is selected out, typically collect density contributions from all atoms in the simulation cell, regardless of how distant they may be from the region of interest. Mulliken population analysis, constructed using the global orbital overlap matrix, has the same problem [30]. In a nutshell, both methods have the fundamental difficulty that the measured population is arbitrary with respect to linear transformations among the selected subset of the orbitals (an example of broken tensorial invariance).

A tensorially invariant population analysis [30,31] instead gathers density contributions and applies constraining potentials only within the region of interest. We will demonstrate here that this can provide very reasonable electronic populations for a physisorbed molecule when using either pseudoatomic orbitals or generalized Wannier functions. Physically motivated orbitals can thus compete with real-space weight functions in cDFT when treated appropriately. Their use may be particularly advantageous in situations in which the system or observable of interest does not readily admit a real-space partitioning, such as when constraining the population of an atom in a crystal, or that of a group of singleparticle states based on their principal angular momentum character.

\section{METHODOLOGY}

\section{A. Constrained density-functional theory forces}

In DFT the ground-state (GS) electron density, $\rho_{0}$, uniquely specifies all the GS properties of a system, including its GS energy [67]. This can thus be found by variationally minimizing an approximate energy functional, $E[\hat{\rho}]$, where $\hat{\rho}$ is the density operator. In cDFT, instead, one seeks to find the GS of the system subject to a constraint, for example the constraint that a given number of electrons is found in a particular subspace. This simple constraint has the mathematical form

$$
\operatorname{Tr}[\hat{\rho} \hat{\mathbb{P}}]-N_{c}=0,
$$

where $\hat{\mathbb{P}}$ is the projection operator for the subspace of interest and $N_{c}$ is the target number of electrons (here "Tr" indicates the trace of the operator, computed over an appropriate basis set). To find the density corresponding to such a constrained ground state, one finds the stationary point $[1,68]$ of the functional $W\left[\hat{\rho}, V_{c}\right]$, where $V_{c}$ is a Lagrange multiplier and

$$
W\left[\hat{\rho}, V_{c}\right]=E[\hat{\rho}]+V_{c}\left(\operatorname{Tr}[\hat{\rho} \hat{\mathbb{P}}]-N_{c}\right) .
$$

For a given $V_{c}$, the Kohn-Sham potential is modified by the addition of the term $V_{c} \hat{\mathbb{P}}$, and $W\left[\hat{\rho}, V_{c}\right]$ is minimized as a functional of $\hat{\rho}$ as usual. Considering just the global minima for each $V_{c}, W$ can be regarded as a function $W\left(V_{c}\right)$ of $V_{c}$ alone [1] (strictly speaking, constrained systems can be constructed where it is a multiple-valued non-function [68]). The stationary points of $W\left(V_{c}\right)$ yield the (potentially degenerate) ground-state densities of the system subject to the given constraint. In particular, the stability of a ground state ensures that $W\left(V_{c}\right)$ attains a maximum [68] with respect to $V_{c}$. At the stationary point $W\left[\hat{\rho}, V_{c}\right]=E[\hat{\rho}]$, since Eq. (5) is satisfied.

In general, $E[\hat{\rho}]$ is not stationary at a nontrivially constrained density, hence the Hellmann-Feynman theorem cannot be applied to $E[\hat{\rho}]$ alone. It is applied instead to $W\left[\hat{\rho}, V_{c}\right]$ in order to find the ionic force

$$
F_{i}=-\frac{d W}{d R_{i}}=-\frac{\partial W}{\partial R_{i}}-\operatorname{Tr}\left[\frac{\partial W}{\partial \hat{\rho}} \frac{d \hat{\rho}}{d R_{i}}\right]-\frac{\partial W}{\partial V_{c}} \frac{d V_{c}}{d R_{i}},
$$

where the index " $i$ " is collective for the ion number and the Cartesian direction indexes. Here the term containing the trace vanishes at any stable ground state by virtue of the HellmannFeynman theorem [68], and the final term vanishes at the cDFT stationary points, i.e., where $\partial W / \partial V_{c}=0$. The force is thus given, in practice, by

$$
F_{i}=-\frac{\partial W}{\partial R_{i}}=-\frac{\partial E[\hat{\rho}]}{\partial R_{i}}-V_{c} \operatorname{Tr}\left[\hat{\rho} \frac{\partial \hat{\mathbb{P}}}{\partial R_{i}}\right] .
$$

The first term on the right-hand side is the contribution from the conventional DFT external potential of the constrained density [69], while the second term, which we will denote by $F_{i}^{c}$, is the Pulay force due to the constraint. Before evaluating this contribution, we must next discuss how the subspace projection operator $\hat{\mathbb{P}}$ is constructed.

\section{B. Tensorially invariant population analysis}

When defining $\hat{\mathbb{P}}$ in terms of nonorthogonal orbitals, such as atomic orbitals centered on atoms, let us label them $\left|\phi_{m}\right\rangle$, 
it is a commonplace and usually unnecessary practice to orthonormalize them by Löwdin transformation. This generates orbitals of the form $\left|\tilde{\phi}_{j}\right\rangle=\sum_{m}\left|\phi_{m}\right\rangle O_{m j}^{-\frac{1}{2}}$, where $\mathbf{O}$ is an orbital overlap matrix. The matrix fractional power is most easily calculated by diagonalizing $\mathbf{O}$, taking the corresponding power of the eigenvalues, and by performing the inverse of the original diagonalizing transformation to arrive at $\mathbf{O}^{-\frac{1}{2}}$.

In methods dealing with the population of orbital-based subspaces such as cDFT and DFT $+U$, it has been shown [30] to be quite incorrect to use, for $\mathbf{O}$, the overlap matrix $\mathbf{S}$ of any larger set that the projector orbitals $\left|\phi_{m}\right\rangle$ may be chosen from, since then the orthonormalized functions $\left|\tilde{\phi}_{j}\right\rangle$ extend across the larger subspace. Instead, if the projection orbitals $\left|\phi_{m}\right\rangle$ used to span a cDFT subspace happen to be selected from a larger set of basis orbitals (e.g., the one spanning the entire Kohn-Sham space), then the subspace overlap matrix $\mathbf{O}$ must be extracted as a sub-block from the full overlap matrix $\mathbf{S}$ before being diagonalized [31].

As an example, let us imagine a bipartite system composed of natural but nontrivially overlapping source and drain regions for a charge-transfer excitation to be accessed using cDFT. If the source-region orbitals $\left|\tilde{\phi}_{j}\right\rangle$ are built using $\mathbf{S}$, then they will extend to some amount over the drain region, and vice versa, in an uncontrolled manner. This pathology will not arise if separate, smaller subspace overlap matrices $\mathbf{O}$ are defined for each of the source and drain regions. This also ensures tensorial invariance and, in particular, physical occupancy eigenvalues (i.e., $0 \leqslant \lambda_{j} \leqslant 1$ ) for the projected density matrices of each constrained subspace [30].

By defining the subspace population as the trace over such orthonormalized functions, we obtain

$$
\begin{aligned}
\operatorname{Tr}[\hat{\rho} \hat{\mathbb{P}}] & =\sum_{j}\left\langle\tilde{\phi}_{j}|\hat{\rho}| \tilde{\phi}_{j}\right\rangle \\
& =\sum_{m n}\left\langle\phi_{m}|\hat{\rho}| \phi_{n}\right\rangle \sum_{j} O_{n j}^{-\frac{1}{2}} O_{j m}^{-\frac{1}{2}} .
\end{aligned}
$$

Equation (8) suggests a straightforward alternative approach, albeit one that is not available if the index $j$ does not run over the same orbital count as $n$ and $m$ (such as when the delocalizing global matrix $\mathbf{S}$ is used). Instead of performing a Löwdin orthonormalization, we may accept the nonorthogonality of the projectors and define the subspace population as a tensor contraction over the nonorthogonal set of $\left|\varphi_{m}\right\rangle$ and their biorthogonal complements $\left|\varphi^{m}\right\rangle$, defined through $\left\langle\varphi^{m} \mid \varphi_{n}\right\rangle=\delta_{n}^{m}$. This gives the transformations

$$
\left|\varphi^{m}\right\rangle=\left|\varphi_{n}\right\rangle O^{n m} \quad \Leftrightarrow \quad\left|\varphi_{m}\right\rangle=\left|\varphi^{n}\right\rangle O_{n m},
$$

where we have adopted the Einstein summation convention for contracting over paired indices, and where $O^{m n}$ is an element of the matrix $\mathbf{O}^{-1}$ with $O_{m n}=\left\langle\varphi_{m} \mid \varphi_{n}\right\rangle$. If the functions $\left|\varphi_{m}\right\rangle$ are chosen to be localized over a particular spatial region, then also the functions $\left|\varphi^{m}\right\rangle$ will be. The required subspace occupancy is then given by

$$
\operatorname{Tr}[\hat{\rho} \hat{\mathbb{P}}]=\operatorname{Tr}\left[\hat{\rho}\left|\varphi^{m}\right\rangle\left\langle\varphi_{m}\right|\right]=\left\langle\varphi_{m}|\hat{\rho}| \varphi_{n}\right\rangle O^{n m},
$$

which is equivalent to Eq. (8). Next, we look at how the Pulay force of cDFT appears when we make this simplification, i.e., when we use the contraction $\sum_{j} O_{n j}^{-\frac{1}{2}} O_{j m}^{-\frac{1}{2}}=O^{n m}$ before the ionic-position derivative is taken.

\section{Nonorthogonality Pulay forces}

A change in the degree of nonorthogonality between projecting orbitals centered on atoms is a natural occurrence in calculations involving ionic displacements. To account for this, the final term of Eq. (7) may be expanded, in view of Eq. (10), as

$$
\begin{aligned}
F_{i}^{c}= & -V_{c}\left[\left\langle\frac{\partial \varphi_{m}}{\partial R_{i}}|\hat{\rho}| \varphi_{n}\right\rangle O^{n m}+\left\langle\varphi_{m}|\hat{\rho}| \frac{\partial \varphi_{n}}{\partial R_{i}}\right\rangle O^{n m}\right. \\
& \left.+\left\langle\varphi_{m}|\hat{\rho}| \varphi_{n}\right\rangle \frac{\partial O^{n m}}{\partial R_{i}}\right] .
\end{aligned}
$$

The first and the second term on the right-hand side represent the force due to the change in the projectors as a result of the ionic displacements, while the third term represents that due to a change in the mutual overlap of the projectors. If the projectors are localized orbitals centered on the atoms, then the third term is exclusively due to the relative motion of the atoms that define the subspace. The first term may be written as $\operatorname{Tr}[\hat{\rho} \hat{X}]$, defining the operator $\hat{X}=\left|\varphi_{n}\right\rangle O^{n m}\left\langle\partial \varphi_{m} / \partial R_{i}\right|$. Similarly, the second term on the right-hand side in Eq. (11) is $\operatorname{Tr}\left[\hat{X}^{\dagger} \hat{\rho}\right]$. For the calculation of this latter term, see Ref. [57].

To evaluate the third term, we shall use the following matrix identity for invertible matrices $\mathbf{M}$ :

$$
\begin{aligned}
\mathbb{D} & =\frac{d}{d R_{i}}[\mathbb{1}]=\frac{d}{d R_{i}}\left[\mathbf{M} \mathbf{M}^{-1}\right]=\frac{d \mathbf{M}}{d R_{i}} \mathbf{M}^{-1}+\mathbf{M} \frac{d \mathbf{M}^{-1}}{d R_{i}} \\
& \Rightarrow \frac{d \mathbf{M}^{-1}}{d R_{i}}=-\mathbf{M}^{-1} \frac{d \mathbf{M}}{d R_{i}} \mathbf{M}^{-1}
\end{aligned}
$$

where $\mathbb{Q}$ is the null matrix. By applying this identity to the overlap matrix $\mathbf{O}$, the third term of Eq. (11) can be rewritten as

$$
\begin{aligned}
& \left\langle\varphi_{m}|\hat{\rho}| \varphi_{n}\right\rangle \frac{\partial O^{n m}}{\partial R_{i}} \\
& =-\left\langle\varphi_{m}|\hat{\rho}| \varphi_{n}\right\rangle O^{n n^{\prime}}\left[\left\langle\frac{\partial \varphi_{n^{\prime}}}{\partial R_{i}} \mid \varphi_{m^{\prime}}\right\rangle+\left\langle\varphi_{n^{\prime}} \mid \frac{\partial \varphi_{m^{\prime}}}{\partial R_{i}}\right\rangle\right] O^{m^{\prime} m} \\
& =-\left\langle\varphi_{n^{\prime}} \mid \frac{\partial \varphi_{m^{\prime}}}{\partial R_{i}}\right\rangle O^{m^{\prime} m}\left\langle\varphi_{m}|\hat{\rho}| \varphi_{n}\right\rangle\left\langle\varphi^{n} \mid \varphi^{n^{\prime}}\right\rangle+\text { c.c. } \\
& =-\operatorname{Tr}\left[\hat{X}^{\dagger} \hat{\rho} \hat{\mathbb{P}}\right]+\text { c.c. }
\end{aligned}
$$

where the projectors obey $\hat{\mathbb{P}}^{\dagger} \hat{\mathbb{P}}=\hat{\mathbb{P}} \hat{\mathbb{P}}=\hat{\mathbb{P}}$.

If we now bring all the terms together, the nonorthogonalityrespecting Pulay force will be given by the remarkably simple expression

$$
\begin{aligned}
F_{i}^{c} & =-V_{c} \operatorname{Tr}\left[\hat{\rho} \hat{X}+\hat{X}^{\dagger} \hat{\rho}-\hat{X}^{\dagger} \hat{\rho} \hat{\mathbb{P}}-\hat{\mathbb{P}} \hat{\rho} \hat{X}\right] \\
& =-2 V_{c} \operatorname{Re} \operatorname{Tr}[\hat{\rho} \hat{X}(\hat{\mathbb{1}}-\hat{\mathbb{P}})] .
\end{aligned}
$$

The final factor, $(\hat{\mathbb{1}}-\hat{\mathbb{P}})$, in this expression is a projector onto the space complementary to the constrained one. The effect of variable nonorthogonality thus becomes clear. It generates 
an extra projection factor that cancels any component of the Pulay force associated with orbital derivatives that are not related to changes in the projected subspace. In other words, it cancels contributions related to changes that cannot cause a variation of the measured occupancy. If the operator $\hat{X}$ applies a linear transformation among the projector orbitals, then $\hat{X}=\hat{X} \hat{\mathbb{P}}$ and the Pulay force will vanish entirely. In contrast, if $\left\langle\partial \varphi_{m} / \partial R_{i} \mid \varphi_{n}\right\rangle=0$ for all $m$ and $n$, then $\hat{X} \hat{\mathbb{P}}=\hat{\mathbb{O}}$ and the expression will reduce to the ordinary Pulay force. It is possible that the projection factor in Eq. (13) is a useful addition to Pulay force calculations in general, since even when orbital nonorthogonality is not expected to arise or vary, numerical noise may cause slight variations from the condition $\left\langle\partial \varphi_{m} / \partial R_{i} \mid \varphi_{n}\right\rangle=0$. An example where this may arise is in force calculations involving atom-centered pseudopotentials defined on a radial grid, which are projected onto a realor reciprocal-space Cartesian grid prior to integration with Kohn-Sham states.

\section{Implementation and procedure for calculation}

We have implemented the nonorthogonality Pulay forces in the linear-scaling DFT code ONETEP [70], which uses strictly localized, variationally optimized nonorthogonal generalized Wannier functions (NGWFs) [28,71,72], $\left|\phi_{\mu}\right\rangle$, as a basis set. The NGWFs are, in turn, expressed as a linear combination of highly localized orthonormal psinc functions, which are essentially Fourier transforms of plane waves specified with a maximum cutoff energy. For a given DFT calculation, ONETEP optimizes the NGWFs using a conjugate-gradient (CG) method in order to minimize the total energy. Within each iteration of such optimization, it minimizes [73] the total-energy functional with respect to the density kernel $K^{\alpha \beta}$, which builds the singleparticle density matrix by means of $\rho\left(\mathbf{r}, \mathbf{r}^{\prime}\right)=\phi_{\alpha}(\mathbf{r}) K^{\alpha \beta} \phi_{\beta}\left(\mathbf{r}^{\prime}\right)$ [73]. Thus, for a geometry optimization in the presence of a constraint of the form contained in Eq. (4), we run the following nested optimization loops:

(i) Optimization of the ionic geometry.

(ii) Conjugate-gradient optimization of the NGWFs $\left|\phi_{\alpha}\right\rangle$ within ensemble DFT.

(iii) Conjugate-gradient optimization of the Lagrange multiplier, $V_{c}$. DFT.

(iv) Optimization of the density kernel $K^{\alpha \beta}$ within ensemble

We note that, although we use the NGWFs as optimized basis functions and as cDFT projectors in this work, the expression for the Pulay forces remains valid for any nonorthogonal set of projector functions. The scheme that we follow for calculating the reorganization energy of a pentacene molecule adsorbed on a flake of graphene can be summarized as follows:

(i) Optimize the geometry of the neutral system and calculate the GS energy with a DFT run. This gives the geometry $G 0$ and the energy $E_{@ G 0}^{0}$.

(ii) Run cDFT for singly ionized pentacene at the geometry $G 0$ in order to obtain the energy $E_{@ G 0}^{+}$.

(iii) Run a constrained geometry optimization to find the nuclear coordinates for the charged pentacene and the corresponding energy. This gives us a geometry $G+$ and an energy $E_{@ G+}^{+}$. (iv) Run DFT on neutral pentacene with geometry $G+$ to find the energy $E_{@ G+}^{0}$ of the neutral configuration at the geometry of the charged state.

The reorganization energy $\lambda$ is then given by

$$
\begin{aligned}
\lambda & =\lambda^{0}+\lambda^{+}, \\
& =\left(E_{@ G+}^{0}-E_{@ G 0}^{0}\right)+\left(E_{@ G 0}^{+}-E_{@ G+}^{+}\right) .
\end{aligned}
$$

Geometry relaxation is performed only on the pentacene molecule, keeping the graphene flake fixed. In other words, the reorganization energies so obtained correspond to pentacene only. Our calculations have been performed with the Perdew-Burke-Ernzerhof (PBE) [74] parametrization of the generalized-gradient approximation of the exchangecorrelation functional and norm-conserving pseudopotentials. The NGWF cutoff radius was set to $9 a_{0}$. It was found that a very high plane-wave cutoff energy of $1500 \mathrm{eV}$ is needed to avoid small changes in energy due to the egg-box effect. cDFT optimization is performed with conjugate gradient with the convergence threshold of $10^{-5} \mathrm{e} / \mathrm{eV}$ for the Lagrange multiplier gradient. This translates to an error of $<4 \times 10^{-4} \%$ in the population of the pentacene molecule. Geometry relaxation is performed with a quasi-Newton method [75] using the Broyden-Fletcher-Goldfarb-Shanno algorithm [76] with Pulay corrected forces (including correction for any residual NGWF nonconvergence [69]) and an energy convergence threshold of $2.5 \times 10^{-6} \mathrm{eV}$ per atom. Some additional features employed in our calculations are described in the following subsections.

A numerical evaluation of the orbitals used to construct the constrained pentacene subspace follows below, but by default we have adopted the well-established practice $[16,22,31,32,77,78]$ of using Wannier functions centered on the appropriate atoms (in this case the pentacene carbon and hydrogen) for the projectors $\left|\varphi_{m}\right\rangle$. In particular, these were chosen as a subset of the NGWFs variationally optimized for the valence manifold of the unconstrained, relaxed neutral ground state of the pentacene-graphene system, following the protocol proposed in Ref. [16] and first applied to cDFT in Ref. [31].

\section{Ensemble density-functional theory}

The occupation number of states in the vicinity of the Fermi level is ill-conditioned in the case of a high degree of degeneracy, as in metals and near-metals like graphene. In other words, significant fluctuations in the occupation numbers and in the electron density take place despite tiny energy changes. In these situations, the number of self-consistent steps necessary for converging the ground state can be large. To circumvent this problem, we employ the finite-temperature ensemble DFT (and cDFT) formalism [79] as implemented within ONETEP [80]. Here, instead of the energy, one minimizes the Helmholtz free energy

$$
\begin{aligned}
A\left[T,\left\{\epsilon_{i}\right\},\left\{\left|\psi_{i}\right\rangle\right\}\right]= & \sum_{i} f_{i}\left\langle\psi_{i}\left|-\frac{1}{2} \nabla^{2}\right| \psi_{i}\right\rangle \\
& +\int d \mathbf{r} v_{n}(\mathbf{r}) \rho(\mathbf{r})+J[\rho]+E_{\mathrm{xc}}\left[\rho^{\alpha}, \rho^{\beta}\right] \\
& -T S\left[\left\{f_{i}\left(\epsilon_{i}\right)\right\}\right],
\end{aligned}
$$


where $S\left[\left\{f_{i}\right\}\right]$ is the entropy of the system given by [81]

$$
S\left[\left\{f_{i}\right\}\right]=-k_{B} \sum_{i}\left[f_{i} \ln f_{i}+\left(1-f_{i}\right) \ln \left(1-f_{i}\right)\right] .
$$

Here, the occupation number $f_{i}\left(\epsilon_{i}\right)$ is that of the $i^{\text {th }} \mathrm{KS}$ state, and it follows the Fermi-Dirac distribution

$$
f_{i}\left(\epsilon_{i}\right)=\left(1-\exp \left[\frac{\epsilon_{i}-\mu}{k_{\mathrm{B}} T}\right]\right)^{-1},
$$

with $\mu$ being the chemical potential, $k_{\mathrm{B}}$ the Boltzmann constant, and $T$ the temperature. In all our calculations, we have used $T=300 \mathrm{~K}$.

\section{Correction for periodic boundary conditions}

Since ONETEP uses the fast Fourier transform to solve the Poisson equation, it requires the use of periodic boundary conditions. For isolated systems one then constructs artificial periodic replica of the simulation cell. This gives rise to undesired interactions between the cells. To correct such a shortcoming, we have used the Martyna-Tuckerman scheme [82] of replacing the Coulomb interaction from the periodic images of the simulation cell with a minimum image convention technique. This essentially adds a screening potential term to approximately cancel the Coulomb interactions from neighboring cells [83]. We used the Martyna-Tuckerman parameter of $7.0 a_{0}$ that is recommended in Ref. [82].

\section{Dispersion correction}

Dispersion interactions, which are poorly accounted for in semilocal exchange and correlation functionals, are expected to be dominant between the pentacene molecule and the graphene flake. Hence, we use an empirical correction, $E_{\mathrm{disp}}\left(r_{i j}\right)$, to the total energy in the form of a damped London term summing over all pairs of atoms $(i, j)$ with an interatomic distance of $r_{i j}$, given by

$$
E_{\mathrm{disp}}\left(r_{i j}\right)=-\sum_{i>j} f_{\mathrm{damp}}\left(r_{i j}\right) \frac{C_{6, i j}}{r_{i j}^{6}},
$$

where $C_{6, i j}$ depends on the particular pair of atoms, and the damping term is given by [84]

$$
f_{\text {damp }}\left(r_{i j}\right)=\left\{1-\exp \left[-c_{\text {damp }}\left(r_{i j} / R_{0, i j}\right)^{7}\right]\right\}^{4} .
$$

The parameters, $c_{\mathrm{damp}}$ and $R_{i j}^{0}$, used here have been generated and implemented previously in the ONETEP code by fitting a set of 60 complexes with significant dispersion [85].

\section{RESULTS}

\section{A. Test of the forces on isolated pentacene}

To demonstrate the role and necessity of using nonorthogonality Pulay corrections, we first present some tests on a very simple system consisting of one isolated, charge-neutral pentacene molecule. We run three independent geometry relaxations:

(i) An unconstrained DFT geometry optimization starting from an idealized initial guess for the ionic geometry of the neutral molecule. This provides a benchmark level of geometry optimization performance on the test system. (ii) A geometry optimization with the same initial guess as (i), while applying a fixed constraint potential of strength $V_{c}$ to the predefined pentacene space and relaxing without the force correction for the derivative of projector overlap [i.e., by omitting the last term on the right-hand side of Eq. (11)].

(iii) The same relaxation as (ii), but including the exact expression of the Pulay forces given in Eq. (11).

A fixed, minimal set of valence pseudo-orbitals (the initial guesses for the NGWFs prior to optimization, i.e., $\mathrm{H} 1 s$ and C $2 p$ and $2 p$ ) was used to define the constrained subspace, with tensorially consistent population analysis. In Fig. 2 we plot the maximum displacement, the change in energy per ion, and the maximum force as a function of the iteration number for the aforementioned calculations performed with two different $V_{c}$, namely 1 and $2.5 \mathrm{eV}$. For $V_{c}=1 \mathrm{eV}$, the three calculations differ only slightly since the force correction is small. However, for $V_{c}=2.5 \mathrm{eV}$ we see that the behavior of the calculation using the incorrect force (red line) differs significantly from the other two, especially for the maximum force on any atom. To quantify the difference in force between the cDFT runs with and without force correction, we calculate the root-meansquared (RMS) difference between the two quantities, given by

$$
\sqrt{\frac{1}{n} \sum_{i}\left(\frac{F_{C}^{i}-F_{U}^{i}}{F_{C}^{i}}\right)^{2}},
$$

where $F_{C}^{i}$ and $F_{U}^{i}$ are, respectively, the corrected and uncorrected total ionic forces on the $i^{\text {th }}$ iteration. Here, $n$ is the total number of iterations, and, clearly, the potential that generates these forces differs except upon the first iteration. The atom with the largest force may also change from iteration to iteration. In percentage terms, the RMS force differences are a very significant $121.73 \%$ and $112.65 \%$ for 1 and $2.5 \mathrm{eV}$ constraint potentials, respectively.

\section{B. Reorganization energy of graphene-adsorbed pentacene}

In this section, we present and discuss our results concerning the reorganization energy of pentacene molecules adsorbed on a flake of graphene. The molecule is positioned above the graphene flake at its center and is oriented parallel to it. We have performed our calculations with two different shapes and sizes of a hydrogen-passivated graphene flake, one containing 358 atoms (hereafter referred to as the smaller flake) and another 474 (hereafter referred to as the larger flake). The geometry of the smaller flake has been relaxed in isolation. However, for the larger flake we use the geometry of an infinite graphene sheet so that the positions of the carbon atoms are symmetric with respect to each other in order to better emulate an infinite graphene sheet. The system is shown in the left-hand-side panel of Fig. 3, while the right-hand-side panel shows a plot of the highest occupied molecular orbital (HOMO) of the entire system. Since the HOMO is mostly localized on the graphene flake (at its edges), simply running a DFT calculation with one less electron is not an option as an electron will be removed from the graphene flake. Thus we use cDFT to constrain a unit positive charge on the pentacene. We emphasize that we do not treat the reorganization effect due to pentacene-graphene charge transfer, but rather the 


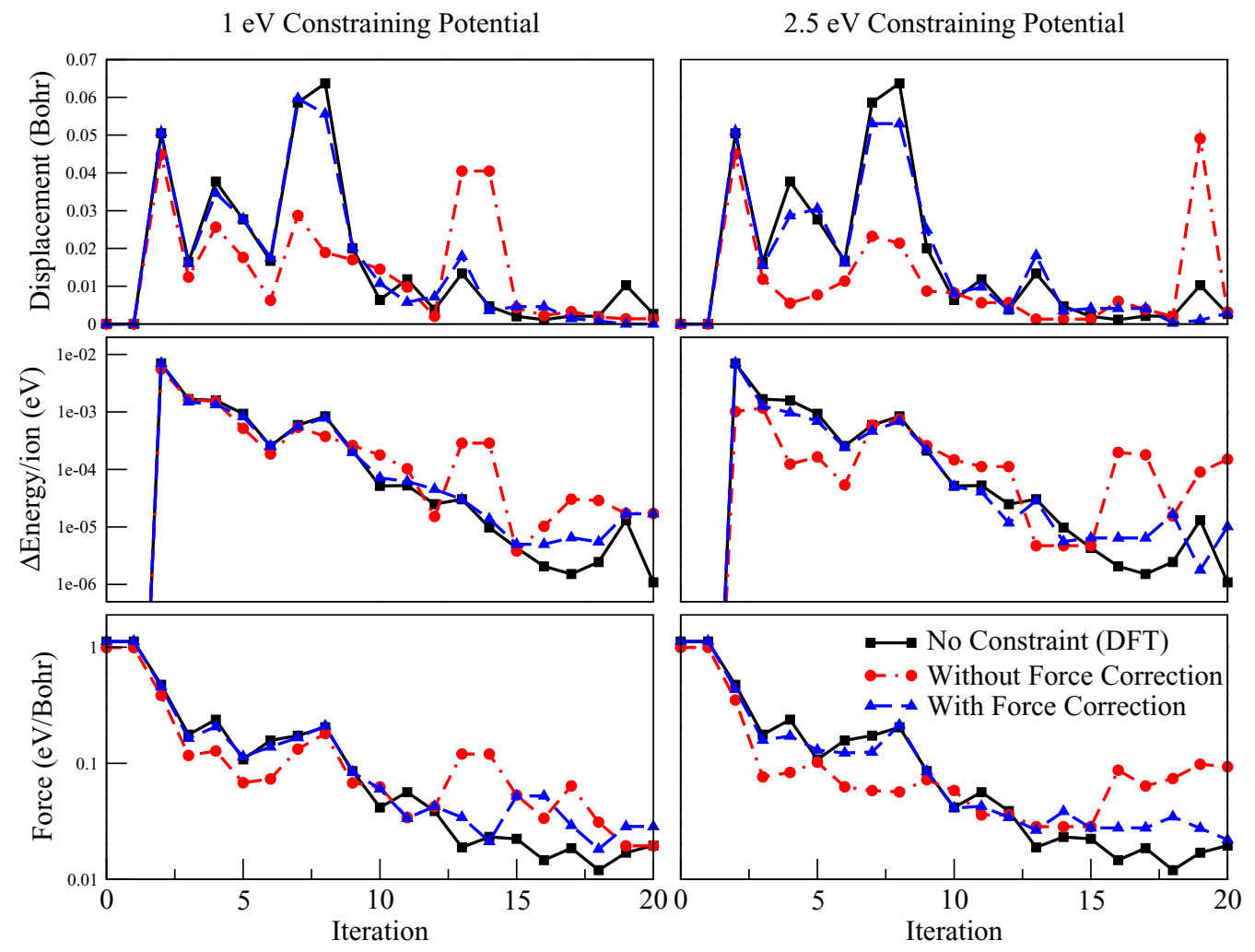

FIG. 2. The maximum displacement of any atom, the change in energy per atom, and the maximum force on any atom, plotted against the iteration number in a geometry relaxation calculation. The black, blue, and red curves show the plots for a regular DFT run, a constrained run without the properly corrected forces, and one with the proper correction for forces, respectively. The constrained calculations are separately performed with a fixed constant potential $V_{c}=1 \mathrm{eV}$ (left column) and $V_{c}=2.5 \mathrm{eV}$ (right column). See the main text for details.

photoemission reorganization effect, in which an electron is removed from the pentacene molecule. This leaves the simulation cell with a net positive charge. The monopole interactions between the periodic replica of the charged unit cells are neutralized by the periodic boundary correction mentioned in Sec. IVD.

\section{Orbital-based population analysis}

In the cDFT calculations, we intend to remove one electron from the pentacene molecule. It is therefore necessary to carry

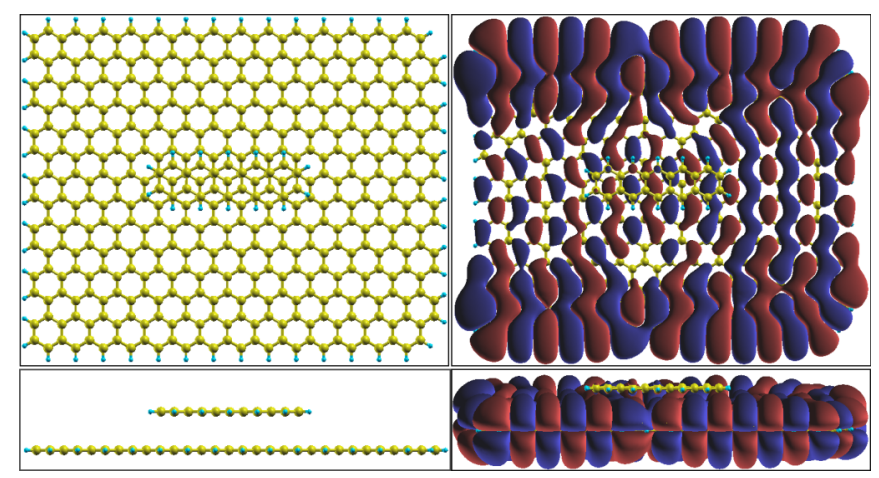

FIG. 3. The left-hand-side panel shows the system of interest, namely a pentacene molecule adsorbed on a graphene flake. The righthand-side panel shows an isovalue plot of the HOMO of the neutral Kohn-Sham system. It is clear that the HOMO is confined to the graphene flake, with most of its amplitude located at its edges. out a population analysis for the uncharged ground state in order to find the number of electrons in the molecule and to define the constraining potential. This population depends on the choice of projectors used to represent the subspace assigned to the molecule. In ONETEP it is possible to use as projectors the atomic pseudo-orbitals (generated from a self-consistent pseudoatomic solver) or the optimized NGWFs from a previous successful run (in our case a DFT run for the same system). In both cases, only the NGWFs associated with the relevant atoms, which here are all the pentacene atoms, are considered. Once the choice of projectors is made, ONETEP allows predominantly two kinds of population analysis on the set of target atoms. The first technique (the "Summed" analysis) essentially calculates the populations on each individual atom and then sums them up. This population is defined as

$$
N_{\text {Summed }}=\sum_{I} \sum_{m m^{\prime}}\left\langle\varphi_{m}^{I}|\hat{\rho}| \varphi_{m^{\prime}}^{I}\right\rangle O_{I}^{m^{\prime} m},
$$

where $I$ is an atom in the desired set and $O_{I}^{m^{\prime} m}$ are the elements of the inverse of the overlap matrix of the projectors $\left|\varphi_{m}^{I}\right\rangle$ and $\left|\varphi_{m^{\prime}}^{I}\right\rangle$ belonging to atom $I$ (this is very close to a Kronecker $\delta$ matrix in the case of the pseudoatomic orbitals). The second one (the "Unified" technique) calculates the tensorially invariant population of the entire subspace as

$$
N_{\text {Unified }}=\sum_{m m^{\prime}}\left\langle\varphi_{m}|\hat{\rho}| \varphi_{m^{\prime}}\right\rangle O^{m^{\prime} m}
$$



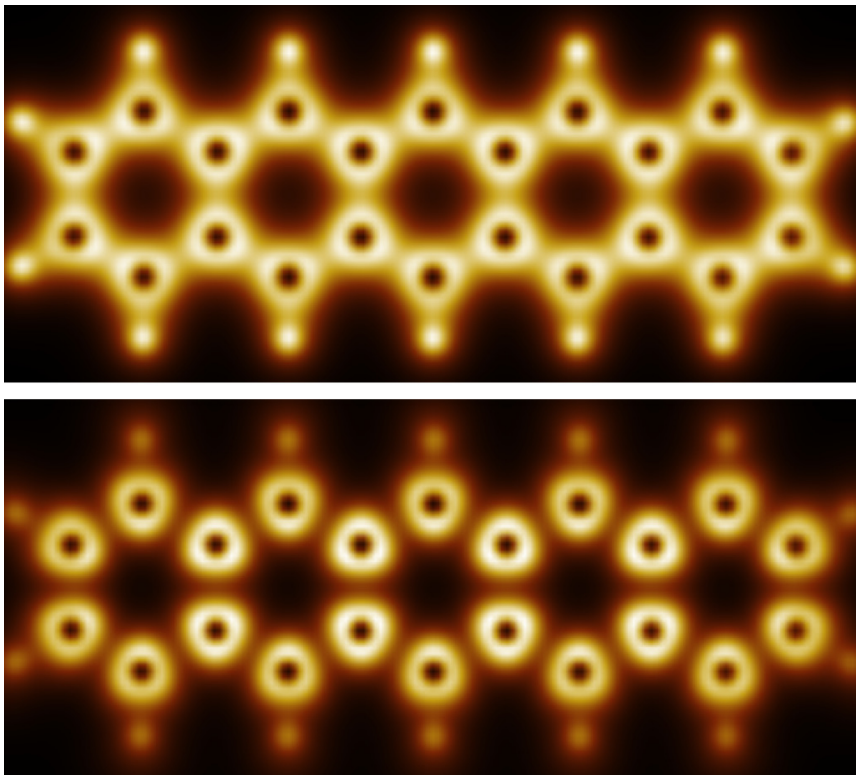

FIG. 4. Plot of $\langle r|\hat{\mathbb{P}}| r\rangle$ for the pentacene molecule adsorbed on graphene in the neutral state. The top and bottom panels correspond, respectively, to the "Summed" analysis, which calculates population on individual atoms separately before adding them up, and the "Unified" analysis, which calculates population of the entire subspace as a whole, respectively. In the case of the Summed method, significant brightness in the interstitial space between atoms indicates doublecounting in the region of orbital overlap. Clearly, this is not the case for the Unified method.

where the sum is over all the orbitals of the given subspace and the inverse overlap matrix is constructed accordingly [30,31]. The "Unified" technique is expected to be much more reliable, since the other double-counts the population shared by the projectors belonging to different atoms. This is clearly seen in Fig. 4, which shows a plot of $\langle r|\hat{\mathbb{P}}| r\rangle$ for the neutral pentacene molecule adsorbed on the graphene flake, where the positions $r$ lie on a plane passing close to all of the pentacene atoms. Using the Summed scheme (top panel), we see significant positive values of $\langle r|\hat{\mathbb{P}}| r\rangle$ in the interstitial region between the atoms, indicating the aforementioned double-counting. As expected, this is not present in the plot for the Unified scheme (bottom panel).

In Table I, we tabulate the populations calculated with the different techniques/projectors on the pentacene molecule, which is adsorbed on a flake of graphene. Noting that an

TABLE I. Number of electrons on the pentacene molecule calculated by using different choices of projectors and for different population analysis methods. An isolated pentacene molecule has 102 valence electrons. See the text in Sec. V B 1 for the definitions of "Summed" and "Unified."

\begin{tabular}{lcc}
\hline \hline Projector & Analysis & Population \\
\hline Atomic orbitals & Summed & 171.56 \\
Atomic orbitals & Unified & 100.74 \\
Optimized NGWFs & Summed & 172.72 \\
Optimized NGWFs & Unified & 102.11 \\
\hline \hline
\end{tabular}
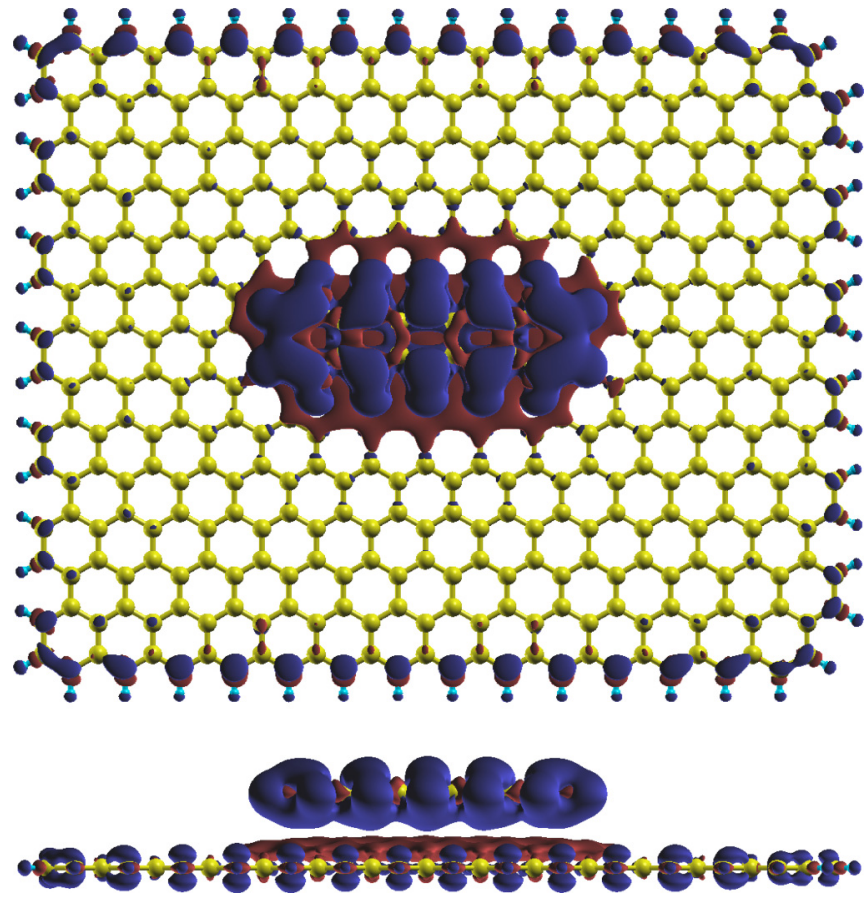

FIG. 5. Plot of isovalues of the change in charge density upon the removal of an electron from the molecule as calculated with cDFT. Blue and red denote positive and negative charge densities, respectively.

isolated pentacene molecule has 102 valence electrons, we see that the combination of optimized NGWFs with the Unified scheme reproduces this count to $0.1 \%$, and so we use this population analysis for further calculations. The residual $0.1 \%$ is due to hybridization with the graphene substrate (a very slight chemisorption effect). We note that pseudoatomic population analysis exhibits an undercount of approximately $1 \%$, but that this is small compared to the error of "Summed," or sometimes known as "on-site," population analysis. The significance of this result is that even pseudoatomic orbitals can provide a reasonable population analysis device for $\mathrm{CDFT}$ if the nonorthogonality or equivalent Löwdin treatment is tensorially invariant (if it uses $\mathbf{O}$ ).

\section{Calculation of the reorganization energy}

Once the population of the molecule, $N$, is determined, the target population for the cDFT calculation is defined as $N_{c}=$ $N \times 101 / 102$. Figure 5 shows the charge density on the system after the removal of an electron from the molecule. As seen in the picture, a molecule with a net positive charge induces a negative charge in the region of the graphene flake immediately beneath the molecule. This is the image charge.

We follow the steps outlined in Sec. IVD to calculate the reorganization energy of the pentacene molecule adsorbed on the graphene flake. Since the final energy of a ONETEP calculation is dependent, albeit very weakly, on the initial NGWFs, we ensure that both the calculations used for computing each instance of $\lambda^{0}$ or $\lambda^{+}$use optimized NGWFs of as similar a provenance as possible. The main problem with such calculation is the existence of multiple configurational local minima differing only slightly in energy. The local minimum to 
which a structural relaxation converges depends largely on the initial geometry. Therefore, we find the reorganization energy corresponding to the two local minima (one for the uncharged system and another for the charged one).

As the opposite image charge formed on the flake results in a Coulomb attraction between the molecule and the flake, in the charged state geometry $G_{+}$the molecule is closer to the flake than in the uncharged geometry $G_{0}$. We also notice that the directions of the in-plane displacements of the atoms of the pentacene molecule upon charging are very similar for the isolated molecule and for the molecule adsorbed on the graphene flake, as can be seen in Fig. 6. Furthermore, the average bond length of the relaxed pentacene molecule is smaller for the charged case, for both the isolated and the adsorbed molecule. This indicates a shrinking of the molecule upon electron emission. Such a change in the average bond length is larger for the isolated pentacene than for the adsorbed one, as indicated by the length of the arrows in Fig. 6. This can be attributed to steric effects due to the presence of graphene. However, as mentioned earlier, one must keep in mind that these properties can, in principle, be specific to the pair of local geometry minima pertaining to the calculation. For a different pair of minima, these values could be different in principle.

In Table II we summarize our results for the reorganization energy for two different cutoff energies and different sizes of the graphene flake. We have also included the reorganization energy of an isolated pentacene molecule (flake =none) for comparison. Note that our results for isolated pentacene matches with that obtained with the MP2 method in an earlier theoretical study [41]. As mentioned in Eq. (3), $\lambda^{0}, \lambda^{+}$, and $\lambda$ refer to the reorganization energy contributions from the uncharged molecule, the positively charged molecule, and the total reorganization energy, respectively.

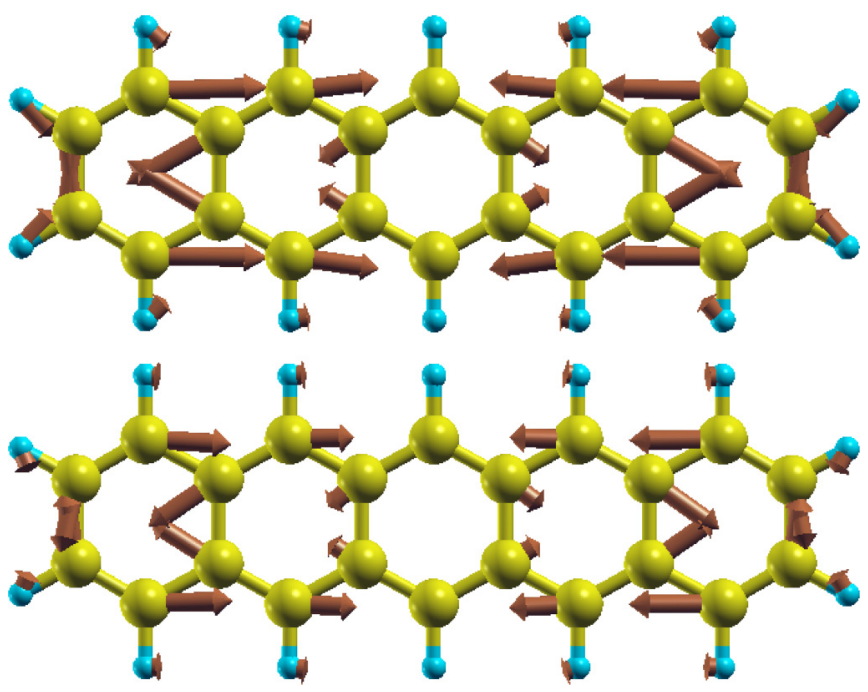

FIG. 6. Pentacene molecular geometry. The arrows show the directions and magnitudes of the in-plane displacement of the atoms in response to the removal of one electron. The top and bottom figures correspond to an isolated pentacene and one deposited on a graphene flake, respectively. The graphene substrate introduces an effective steric hindrance, which reduces the reorganization effect and energy.
TABLE II. Reorganization energies (corresponding to local minima in the geometry) of a pentacene molecule as a function of the cutoff energy and the size of the graphene flake. $\Delta V_{c}$ denotes the difference in the cDFT Lagrange multipliers corresponding to the two different geometries. All energies are in meV. The smaller and the larger flakes contain 358 and 474 atoms at the optimized and idealized positions, respectively.

\begin{tabular}{lccccc}
\hline \hline Cutoff energy (eV) & Flake & $\lambda^{0}$ & $\lambda^{+}$ & $\lambda$ & $\Delta V_{c}$ \\
\hline 900 & none & 29 & 27 & 56 & N.A. \\
900 & smaller & 23 & 26 & 49 & 44 \\
900 & larger & 20 & 20 & 39 & 50 \\
1500 & none & 29 & 27 & 56 & N.A. \\
1500 & smaller & 25 & 25 & 51 & 45 \\
1500 & larger & 17 & 23 & 40 & 33 \\
\hline \hline
\end{tabular}

Since the reorganization energy is very small in general, minute fluctuations (per atom) arising due to diverse local geometry minima or differences in the NGWF initial state can change the results considerably. As a result of exhaustive calculations using different NGWF restart protocols, we estimate the root-mean-square value of error caused by such deviations to be approximately $6 \mathrm{meV}$ for each instance of $\lambda^{0}$ or $\lambda^{+}$. Therefore, in Table II, we focus predominantly on the general trend in the results, which we consider to be quite robust, rather than the precise values. A surprising effect to observe here is that the total reorganization energy $\lambda$ appears to be insensitive to changes in the kinetic cutoff energy, relative to its separate components $\lambda^{0}$ and $\lambda^{+}$. It is not possible to conclude that this is more generally the case based on the available evidence. The take-home message of the table is that the reorganization energy of the isolated molecule is generally greater than that of the same molecule on graphene. This can be attributed to steric effects for the latter case, namely to the fact that an adsorbed molecule has less freedom for ionic relaxation.

The reorganization energy is lower for the larger flake. We attribute this to two possible mechanisms: (i) the freedom of ionic motion of the molecule may be more restricted for a larger substrate; (ii) since, as mentioned earlier, the bond lengths in the smaller flake are not all equal, adsorption on this flake is likely to result in a more uneven energy landscape for the pentacene molecule. It is worth noting that we have analyzed the different contributions due to Hartree, exchange and correlation, pseudopotentials, and kinetic energy to the reorganization energy. However, the relatively small reorganization energy turns out to be the result of the substantial cancellation of large variations in these individual terms. It is noteworthy that experimental studies $[62,63]$ on a rather different system of graphene-adsorbed pentacene, namely a thin film of pentacene deposited on HOPG, conversely exhibits an increase in reorganization energy with respect to the isolated pentacene molecule. This points to the possibility that intermolecular relaxation in the film contributes to the reorganization energy and more than compensates for the effects of steric hindrance.

Here we note that, since, strictly speaking, the polarizability of the neutral molecule is different from that of the charged one, using the same form of empirical vdW correction for the molecule-flake interface in both cases may introduce some 
bias in the numerical results. To obtain an estimate for such error, we calculate, without using any vdW correction, the reorganization energy of pentacene adsorbed on the smaller flake using a plane-wave cutoff of $900 \mathrm{eV}$. We see that the results so obtained $\left(\lambda^{0}=23 \mathrm{meV}\right.$ and $\left.\lambda^{+}=29 \mathrm{meV}\right)$ are similar to those obtained with vdW corrections, and that the difference is within the range of fluctuations caused by local minima and in the NGWF restart protocol. We infer that the inclusion of the vdW corrections does not alter the reorganization energy significantly.

We finally note that the Lagrange multiplier $V_{c}$ for oneelectron removal may be interpreted as an unscreened approximate subspace-local ionization potential, and that the extent of the screening may be assumed to be independent of small changes in the ionic geometry. Therefore, the difference, $\Delta V_{c}$, between the converged Lagrange multipliers for the charged pentacene in geometries $G_{0}$ and $G_{+}$can be taken as an approximation for the reorganization energy. Also, since this quantity is evaluated explicitly only on the basis of the occupancy of the adsorbate, we may expect it to be relatively free (that is, compared to the true reorganization energy) from numerical errors in the optimized ionic positions of distant atoms in the graphene flake. Consequently, in Table II, we find that $\Delta V_{c}$ is slightly less dependent on the nature of the substrate than the true reorganization energy is, but, in contrast, it seems to be too sensitive to the plane-wave energy cutoff for practical utility.

\section{CONCLUSION}

We have presented a method for calculating self-consistent forces in conjunction with constrained DFT in first-principles calculations employing atom-centered functions. We have investigated a very accurate population analysis constructed over Wannier functions and a tensorially consistent treatment of nonorthogonality. This is shown to yield an exact expression for force containing a Pulay term for the change in nonorthogonality, which circumvents the need for overlap matrix diagonalization and is compatible with complex-valued orbitals. We have implemented this expression for the force in the DFT code ONETEP and have shown that the contribution to the force arising from the change in mutual overlap of the nonorthogonal projector orbitals of the subspace exerts significant influence on the geometry relaxation.

To show a novel practical application of such forces, we perform a hyperaccurate geometry optimization with numerous extra features to capture the reorganization energy of a pentacene molecule adsorbed on a flake of graphene. We have argued that the Lagrange multiplier itself can be used to provide a local estimate of the reorganization energy in systems in which the principal change to the system is spatially localized. Since the geometry of such a system has multiple local minima closely related in energy, the reorganization energy can, in principle, be calculated only over such local minima. These depend on the initial geometry. We show that for the minima obtained in our calculations, the reorganization energy of the molecule adsorbed on a graphene flake is typically smaller than that of the isolated molecule, a fact that is consistent with a steric hindrance effect.

\section{ACKNOWLEDGMENTS}

This work is supported by the European Research Council project QUEST (Grant No. 307891). We acknowledge and thank G. Teobaldi and N. D. M. Hine for their implementation and automation of cDFT in ONETEP, D. Turban for extending the population analysis to encompass orbitals across multiple atomic centers, and C.-K. Skylaris and his team for their prior implementation of the dispersion correction, boundary condition correction, and ensemble DFT in that package. The authors acknowledge the DJEI/DES/SFI/HEA Irish Centre for High-End Computing (ICHEC) for the provision of computational facilities and support. We also acknowledge Trinity Research IT for the maintenance of the Boyle cluster on which further calculations were performed.
[1] Q. Wu and T. van Voorhis, Phys. Rev. A 72, 024502 (2005).

[2] B. Kaduk, T. Kowalczyk, and T. van Voorhis, Chem. Rev. 112, 321 (2012).

[3] V. I. Anisimov, J. Zaanen, and O. K. Andersen, Phys. Rev. B 44, 943 (1991).

[4] B. Himmetoglu, A. Floris, S. de Gironcoli, and M. Cococcioni, Int. J. Quantum Chem. 114, 14 (2014).

[5] V. I. Anisimov, A. I. Poteryaev, M. A. Korotin, A. O. Anokhin, and G. Kotliar, J. Phys. Condens. Matter 9, 7359 (1997).

[6] G. Kotliar, S. Y. Savrasov, K. Haule, V. S. Oudovenko, O. Parcollet, and C. A. Marianetti, Rev. Mod. Phys. 78, 865 (2006).

[7] S. J. Bennie, M. Stella, T. F. Miller, and F. R. Manby, J. Chem. Phys. 143, 024105 (2015).

[8] P. Sherwood, A. H. de Vries, S. J. Collins, S. P. Greatbanks, N. A. Burton, M. A. Vincent, and I. H. Hillier, Faraday Discuss. 106, 79 (1997).

[9] L. P. Lee, D. J. Cole, M. C. Payne, and C.-K. Skylaris, J. Comput. Chem. 34, 429 (2013).

[10] N. Marzari and D. Vanderbilt, Phys. Rev. B 56, 12847 (1997).
[11] L. Peng, F. L. Gu, and W. Yang, Phys. Chem. Chem. Phys. 15, 15518 (2013).

[12] O. K. Andersen, T. Saha-Dasgupta, R. W. Tank, C. Arcangeli, O. Jepsen, and G. Krier, in Electronic Structure and Physical Properties of Solids. The Use of the LMTO Method, edited by H. Dreyssé, Lecture Notes in Physics Vol. 535 (Springer-Verlag, Berlin, 2000), p. 3.

[13] F. Weinhold and C. R. Landis, Chem. Ed. Res. Pract. 2, 91 (2001).

[14] M. Soriano and J. J. Palacios, Phys. Rev. B 90, 075128 (2014).

[15] D. Jacob, J. Phys. Condens. Matter 27, 245606 (2015).

[16] D. D. O'Regan, N. D. M. Hine, M. C. Payne, and A. A. Mostofi, Phys. Rev. B 82, 081102 (2010).

[17] Y.-C. Wang, Z.-H. Chen, and H. Jiang, J. Chem. Phys. 144, 144106 (2016).

[18] K. Haule, C.-H. Yee, and K. Kim, Phys. Rev. B 81, 195107 (2010).

[19] P. Pulay, G. Fogarasi, F. Pang, and J. E. Boggs, J. Am. Chem. Soc. 101, 2550 (1979). 
[20] M. Methfessel and M. van Schilfgaarde, Phys. Rev. B 48, 4937 (1993).

[21] Q. Wu and T. van Voorhis, J. Phys. Chem. A 110, 9212 (2006).

[22] D. Novoselov, D. M. Korotin, and V. I. Anisimov, J. Phys. Condens. Matter 27, 325602 (2015).

[23] R. H. Bartels and G. W. Stewart, Commun. ACM 15, 820 (1972).

[24] R. S. Mulliken, J. Chem. Phys. 23, 1833 (1955).

[25] E. B. Stechel, T. G. Schmalz, and J. C. Light, J. Chem. Phys. 70, 5640 (1979).

[26] M. Head-Gordon, P. E. Maslen, and C. A. White, J. Chem. Phys. 108, 616 (1998).

[27] E. Artacho and L. Miláns del Bosch, Phys. Rev. A 43, 5770 (1991).

[28] C.-K. Skylaris, A. A. Mostofi, P. D. Haynes, O. Diéguez, and M. C. Payne, Phys. Rev. B 66, 035119 (2002).

[29] E. Artacho and D. D. O’Regan, Phys. Rev. B 95, 115155 (2017).

[30] D. D. O'Regan, M. C. Payne, and A. A. Mostofi, Phys. Rev. B 83, 245124 (2011).

[31] D. H. P. Turban, G. Teobaldi, D. D. O'Regan, and N. D. M. Hine, Phys. Rev. B 93, 165102 (2016).

[32] S. Lukman, K. Chen, J. M. Hodgkiss, D. H. P. Turban, N. D. M. Hine, S. Dong, J. Wu, N. C. Greenham, and A. J. Musser, Nat. Commun. 7, 13622 (2016).

[33] R. A. Marcus, J. Electroanal. Chem. 438, 251 (1997).

[34] P. F. Barbara, T. J. Meyer, and M. A. Ratner, J. Phys. Chem. 100, 13148 (1996).

[35] V. Rühle, A. Lukyanov, F. May, M. Schrader, T. Vehoff, J. Kirkpatrick, B. Baumeier, and D. Andrienko, J. Chem. Theory Comput. 7, 3335 (2011).

[36] F. Manke, J. M. Frost, V. Vaissier, J. Nelson, and P. R. F. Barnes, Phys. Chem. Chem. Phys. 17, 7345 (2015).

[37] S. Jakobsen, K. V. Mikkelsen, and S. U. Pedersen, J. Phys. Chem 100, 7411 (1996).

[38] H.-S. Ren, M.-J. Ming, J.-Y. Ma, and X.-Y. Li, J. Phys. Chem. A 117, 8017 (2013).

[39] Y. Yamashita, Sci. Technol. Adv. Mater. 10, 024313 (2009).

[40] T. Hasegawa and J. Takeya, Sci. Tech. Adv. Mater. 10, 024314 (2009).

[41] N. E. Gruhn, D. A. da Silva Filho, T. G. Bill, M. Malagoli, V. Coropceanu, A. Kahn, and J. L. Brédas, J. Am. Chem. Soc. 124, 7918 (2002).

[42] V. Coropceanu, M. Malagoli, D. A. da Silva Filho, N. E. Gruhn, T. G. Bill, and J. L. Brédas, Phys. Rev. Lett. 89, 275503 (2002).

[43] R. S. Sánchez-Carrera, V. Coropceanu, D. A. da Silva Filho, R. Friedlein, W. Osikowicz, R. Murdey, C. Suess, W. R. Salaneck, and J.-L. Brédas, J. Phys. Chem. B 110, 18904 (2006).

[44] V. Coropceanu, R. S. Sánchez-Carrera, P. Paramonov, G. M. Day, and J.-L. Brédas, J. Phys. Chem. C 113, 4679 (2009).

[45] V. Coropceanu, J. Cornil, D. A. da Silva Filho, Y. Olivier, R. Silbey, and J.-L. Brédas, Chem. Rev. 107, 926 (2007).

[46] A. Troisi, Chem. Soc. Rev. 40, 2347 (2011).

[47] X. Yang, L. Wang, C. Wang, W. Long, and Z. Shuai, Chem. Mater. 20, 3205 (2008).

[48] J.-H. Franke, N. N. Nair, L. Chi, and H. Fuchs, Constrained density functional theory of molecular dimers, in High Performance Computing in Science and Engineering '11: Transactions of the High Performance Computing Center, Stuttgart (HLRS), 2011, edited by W. E. Nagel, D. B. Kröner, and M. M. Resch (Springer, Berlin, 2012), pp. 169-183.
[49] M. Melander, E. O. Jónsson, J. J. Mortensen, T. Vegge, and J. M. García Lastra, J. Chem. Theory Comput. 12, 5367 (2016).

[50] H. Nakano and H. Sato, J. Chem. Phys. 146, 154101 (2017).

[51] L. E. Ratcliff, L. Grisanti, L. Genovese, T. Deutsch, T. Neumann, D. Danilov, W. Wenzel, D. Beljonne, and J. Cornil, J. Chem. Theory Comput. 11, 2077 (2015).

[52] K. M. Pelzer, A. Vazquez-Mayagoitia, L. E. Ratcliff, S. Tretiak, R. A. Bair, S. K. Gray, T. van Voorhis, R. E. Larsen, and S. B. Darling, Chem. Sci. 8, 2597 (2017).

[53] A. Droghetti, I. Rungger, C. Das Pemmaraju, and S. Sanvito, Phys. Rev. B 93, 195208 (2016).

[54] N. Gillet, L. Berstis, X. Wu, F. Gajdos, A. Heck, A. de la Lande, J. Blumberger, and M. Elstner, J. Chem. Theor. Comput. 12, 4793 (2016).

[55] N. Holmberg and K. Laasonen, J. Chem. Theor. Comput. 13, 587 (2017).

[56] N. Davari, P. O. Åstrand, and T. V. Voorhis, Mol. Phys. 111, 1456 (2013).

[57] D. D. O'Regan, N. D. M. Hine, M. C. Payne, and A. A. Mostofi, Phys. Rev. B 85, 085107 (2012).

[58] A. M. Souza, I. Rungger, C. D. Pemmaraju, U. Schwingenschloegl, and S. Sanvito, Phys. Rev. B 88, 165112 (2013).

[59] S. Roychoudhury, C. Motta, and S. Sanvito, Phys. Rev. B 93, 045130 (2016).

[60] S. Kirkpatrick, C. D. Gelatt, and M. P. Vecchi, Science 220, 671 (1983).

[61] D. J. Wales and J. P. K. Doye, J. Phys. Chem. A 101, 5111 (1997).

[62] S. Kera, H. Yamane, and N. Ueno, Prog. Surf. Sci. 84, 135 (2009).

[63] H. Yamane, S. Nagamatsu, H. Fukagawa, S. Kera, R. Friedlein, K. K. Okudaira, and N. Ueno, Phys. Rev. B 72, 153412 (2005).

[64] P. B. Paramonov, V. Coropceanu, and J.-L. Brédas, Phys. Rev. B 78, 041403 (2008).

[65] C. Tablero, J. Phys. Condens. Matter 20, 325205 (2008).

[66] M. J. Han, T. Ozaki, and J. Yu, Phys. Rev. B 73, 045110 (2006).

[67] P. Hohenberg and W. Kohn, Phys. Rev. 136, B864 (1964).

[68] D. D. O'Regan and G. Teobaldi, Phys. Rev. B 94, 035159 (2016).

[69] A. Ruiz-Serrano, N. D. M. Hine, and C.-K. Skylaris, J. Chem. Phys. 136, 234101 (2012).

[70] C.-K. Skylaris, P. D. Haynes, A. A. Mostofi, and M. C. Payne, J. Chem. Phys. 122, 084119 (2005).

[71] A. A. Mostofi, C.-K. Skylaris, P. D. Haynes, and M. C. Payne, Comput. Phys. Commun. 147, 788 (2002).

[72] D. D. O’Regan, M. C. Payne, and A. A. Mostofi, Phys. Rev. B 85, 193101 (2012).

[73] P. D. Haynes, C.-K. Skylaris, A. A. Mostofi, and M. C. Payne, J. Phys. Condens. Matter 20, 294207 (2008).

[74] J. P. Perdew, K. Burke, and M. Ernzerhof, Phys. Rev. Lett. 77, 3865 (1996).

[75] B. G. Pfrommer, M. Côté, S. G. Louie, and M. L. Cohen, J. Comput. Phys. 131, 233 (1997).

[76] N. D. M. Hine, M. Robinson, P. D. Haynes, C.-K. Skylaris, M. C. Payne, and A. A. Mostofi, Phys. Rev. B 83, 195102 (2011).

[77] D. Korotin, V. Kukolev, A. V. Kozhevnikov, D. Novoselov, and V. I. Anisimov, J. Phys. Condens. Matter 24, 415603 (2012).

[78] F. Lechermann, A. Georges, A. Poteryaev, S. Biermann, M. Posternak, A. Yamasaki, and O. K. Andersen, Phys. Rev. B 74, 125120 (2006).

[79] N. Marzari, D. Vanderbilt, and M. C. Payne, Phys. Rev. Lett. 79, 1337 (1997). 
[80] A. Ruiz-Serrano and C.-K. Skylaris, J. Chem. Phys. 139, 054107 (2013).

[81] F. Mandl, Statistical Physics, Manchester Physics Series (Wiley, Chichester, 1971).

[82] G. J. Martyna and M. E. Tuckerman, J. Chem. Phys. 110, 2810 (1999).
[83] N. D. M. Hine, J. Dziedzic, P. D. Haynes, and C.-K. Skylaris, J. Chem. Phys. 135, 204103 (2011).

[84] M. Elstner, P. Hobza, T. Frauenheim, S. Suhai, and E. Kaxiras, J. Chem. Phys. 114, 5149 (2001).

[85] Q. Hill and C.-K. Skylaris, Proc. R. Soc. A 465, 669 (2009). 